\title{
Bolus administration T1 mapping as a marker of interstitial myocardial fibrosis in severe aortic stenosis
}

\author{
Rick Wage ${ }^{*}$, Andrew Jabbour, Tevfik F Ismail \\ From 15th Annual SCMR Scientific Sessions \\ Orlando, FL, USA. 2-5 February 2012
}

\begin{abstract}
Summary
Post-gadolinium bolus T1 mapping was used to assess interstitial myocardial fibrosis in a group of patients with severe aortic stenosis. The changes identified were correlated with regional contractile perfomance as assessed by CSPAMM tagging. T1 mapping proved to be a practical feasible approach for assessing diffuse fibrosis.
\end{abstract}

\section{Background}

Aortic stenosis (AS) is associated with increased interstitial myocardial fibrosis (IMF). This is detectable by cardiovascular magnetic resonance (CMR) after gadolinium infusion. T1-mapping-derived partition coefficients $(\lambda)$ and extracellular volume fractions $(\mathrm{Ve})$ have also been shown to correlate with IMF after a simple bolus administration of gadolinium. We hypothesized that the $\lambda$ and Ve would also detect interstitial expansion in severe AS patients compared to healthy controls and that these measures would correlate with abnormal myocardial strain using a high temporal-resolution tagging sequence.

\section{Methods}

A Modified Look-Locker Inversion Recovery (MOLLI) sequence was used to generate eleven T1-weighted images. Myocardial and blood pool T1 values were derived by fitting a signal intensity-time curve using CMR $42^{\circledR}$. The $\lambda$ was determined by plotting (1/T1myo vs. 1/T1blood pool) at various time points once contrast equilibrium was reached. Ve was derived accounting for the hematocrit. Ventricular long-axis and short-axis T1 maps (basal, mid-ventricular and apical) were acquired using a $1.5 \mathrm{~T}$ scanner (Siemens) before and $1,2,5,8,15,20,25$ and 30 minutes after contrast. Myocardial tagging images were acquired using both singleand multiple-breath-hold Complementary Spatial Modulation of Magnetization (CSPAMM) sequences in multiple planes and analysed with inTag ${ }^{\circledR}$ (Lyon, France).

\section{Results}

Subjects with severe AS displayed higher $\lambda$ and Ve $(\mathrm{p}=0.02)$. The $\lambda$ and Ve correlated with indices of reduced myocardial function including reduced strain $(\mathrm{p}<0.05)$ and increased left atrial dilatation $(\mathrm{p}=0.001)$. In this presentation, the tips and pitfalls of $\mathrm{T} 1$ mapping using MOLLI will be discussed, including detailed discussion of imaging planes, arrhythmia management, breath-hold times, gadolinium administration and artefact reduction.

\section{Conclusions}

T1-mapping-derived $\lambda$ and Ve are significantly elevated in patients with AS compared to healthy controls and correlate well with indices of reduced myocardial performance. This difference was quantifiable after a simple bolus administration of gadolinium. T1-mapping $\lambda$ and Ve derivation after bolus gadolinium administration is clinically practical and holds promise for the detection of IMF in severe AS.

\section{Funding}

This project was supported by the NIHR Royal Brompton Cardiovascular Biomedical Research Unit.

Published: 1 February 2012

CMR Unit, Royal Brompton Hospital, London, UK

(c) 2012 Wage et al; licensee BioMed Central Ltd. This is an open access article distributed under the terms of the Creative Commons Attribution License (http://creativecommons.org/licenses/by/2.0), which permits unrestricted use, distribution, and reproduction in any medium, provided the original work is properly cited. 
doi:10.1186/1532-429X-14-S1-T4

Cite this article as: Wage et al:: Bolus administration T1 mapping as a

marker of interstitial myocardial fibrosis in severe aortic stenosis. Journal

of Cardiovascular Magnetic Resonance 2012 14(Suppl 1):T4.

Submit your next manuscript to BioMed Central and take full advantage of:

- Convenient online submission

- Thorough peer review

- No space constraints or color figure charges

- Immediate publication on acceptance

- Inclusion in PubMed, CAS, Scopus and Google Scholar

- Research which is freely available for redistribution

Submit your manuscript at 